\title{
Le syndrome hématopoiétique induit par une irradiation accidentelle : évaluation et traitements possibles
}

\author{
J.-M. BERTHO ${ }^{1}$
}

(Manuscrit reçu le 9 septembre 2005, accepté le 7 janvier 2006)

RÉSUMÉ Le syndrome hématopoiétique induit par une irradiation accidentelle reste difficile à traiter, essentiellement du fait de l'hétérogénéité de l'irradiation. La conséquence est qu'il y a toujours un territoire médullaire partiellement protégé de l'irradiation. Le choix d'une stratégie thérapeutique va dépendre essentiellement de l'évaluation des dommages radio-induits à la moelle osseuse, telle qu'elle peut être faite à partir de différents bio-indicateurs de dose ou de dommages comme le suivi de la concentration sanguine de Flt3 ligand. Les options thérapeutiques restent limitées à la greffe de cellules souches hématopoïétiques ou aux traitements de soutien. Récemment, de nouvelles approches de thérapie cellulaire autologue ont été proposées, et ont apporté un éclairage nouveau sur le syndrome de défaillance multiviscérale radio-induit. D'autres résultats récents du laboratoire montrent que parmi les choix possibles, la stimulation de l'hématopoìèse résiduelle par l'injection de cytokines telles que le G-CSF reste l'une des options thérapeutiques les plus intéressantes. Ces donnés nouvelles soulignent l'importance de la notion d'hématopoï̀se résiduelle, mais aussi le fait que les mécanismes de régulation de l'hématopoièse résiduelle après irradiation restent mal connus.

ABSTRACT The hematopoietic syndrome induced by an accidental radiation: evaluation and treatments.

The accidental radiation-induced hematopoietic syndrome remains difficult to treat, mainly due to the heterogeneity of irradiation. As a result, there always remains an area of active hematopoiesis that was partly protected from irradiation. The choice of a therapeutic strategy must be based upon the estimate of radiation damage to the bone marrow. This can be achieved by the use of biological indicators of radiation dose or damage such as the follow up of Flt3 ligand concentration in the blood. The possible therapeutic strategies remain limited, either stem cell transplantation or supportive care. A new approach based upon autologous cell therapy was proposed and the results highlighted new insight about the radiation-induced multiple organ disease syndrome. Other recent results from our lab suggested that among the possible therapeutic strategies, the stimulation of residual hematopoesis by the mean of cytokines such as G-CSF is one of the most interesting strategies. These new data highlight the notion of residual hematopoiesis but also the fact that regulation mechanisms of residual hematopoiesis are not well understood.

Key words: acute exposure / radiation damage / bone marrow / biological indicator / treatment

\footnotetext{
1 IRSN, DRPH/SRBE, Laboratoire de thérapie cellulaire et de radioprotection accidentelle, 92262 Fontenay-aux-roses Cedex, France.
} 


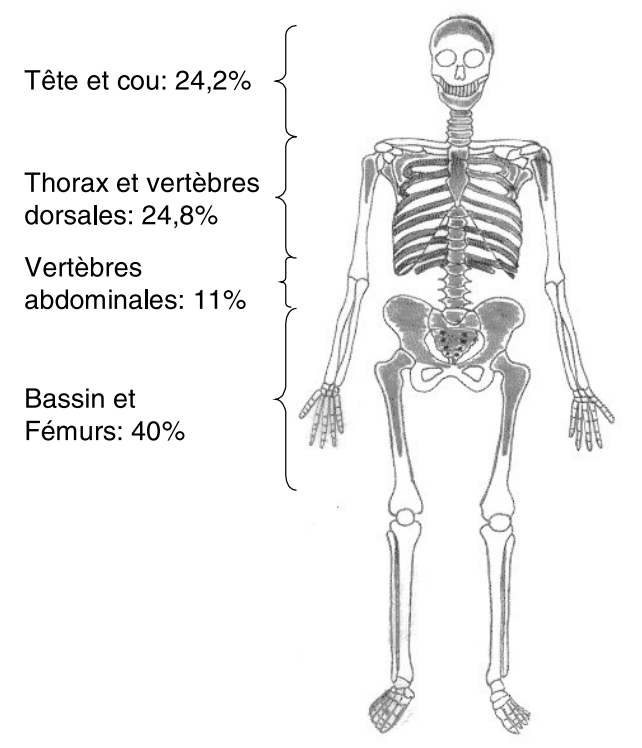

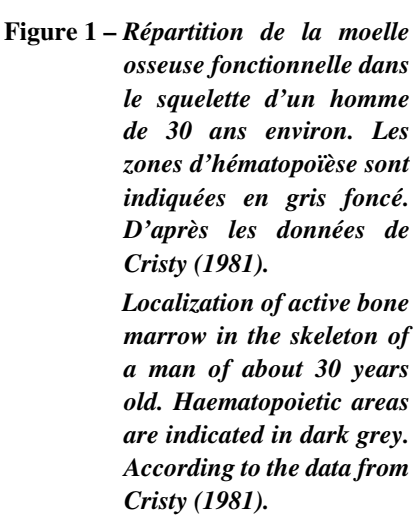

\section{La nature hétérogène de l'irradiation accidentelle}

Les accidents d'irradiation globale à forte dose, bien que rares, posent toujours des problèmes thérapeutiques importants, avec une issue fatale pour la plupart des victimes. Ce mauvais pronostic est essentiellement dû à deux facteurs, d'une part une physiopathologie des irradiations complexes mal connue, et d'autre part la nature hétérogène des irradiations accidentelles. Les accidents d'irradiation récents, et notamment l'accident de Tokai-mura (Kanda et al., 2002), ont montré que le choix d'une stratégie thérapeutique ne doit plus être basé uniquement sur l'estimation de la dose reçue, mais aussi sur l'estimation des dommages aux systèmes physiologiques vitaux, particulièrement lorsqu'une irradiation hétérogène est soupçonnée. Cette démarche est cruciale pour le syndrome hématopoïétique, qui est dû à la destruction partielle ou totale des cellules souches de la moelle osseuse. La moelle osseuse n'est alors plus capable d'assurer sa fonction de renouvellement des cellules sanguines. Ceci entraîne la disparition progressive des leucocytes, des plaquettes et des globules rouges de la circulation sanguine. Il en résulte des risques infectieux et hémorragiques importants, pouvant mettre en jeu le pronostic vital des victimes d'irradiation accidentelle. Néanmoins, il faut tenir compte de la répartition de la moelle osseuse dans l'ensemble de l'organisme, essentiellement dans les os long (Fig. 1) (Cristy, 1981). Cette répartition anatomique, associée à la 
nature hétérogène des irradiations accidentelles, fait que dans la plupart des cas, il persiste toujours un territoire médullaire au moins partiellement protégé de l'irradiation. Ainsi, la reconstruction dosimétrique de l'accident de Mol (Belgique en 1965) où un opérateur s'est appuyé sur un assemblage de combustible, a démontré l'existence de territoires médullaires irradiés à des doses inférieures à 3 Gy à partir desquels une reprise hématopoiétique a été observée (Parmentier et al., 1980). L'existence de cellules souches hématopoïétiques (CSH) survivantes à plus de 10 Gy a été démontrée chez la victime de l'accident de Nesvizh (Biélorussie en 1991 ; Baranov et al., 1994), et le rejet de la greffe de CSH chez l'un des trois irradiés de Tokai-mura est une démonstration de l'existence d'une hématopoïèse résiduelle, suffisante pour assurer une reconstitution autologue après une irradiation accidentelle à forte dose (Nagayama et al., 2002).

Ces constatations montrent que deux questions cruciales se posent face à une irradiation accidentelle : d'une part, l'estimation de la sévérité des dommages à la moelle osseuse en tenant compte de l'hétérogénéité de l'irradiation, et d'autre part le choix de la stratégie thérapeutique à appliquer en fonction de cette évaluation de l'atteinte à la moelle osseuse.

\section{L'évaluation des dommages radio-induits au système hématopoïétique}

L'évaluation des dommages radio-induits est restée longtemps basée sur la dose moyenne à l'organisme, mesurée a posteriori par des méthodes physiques ou biologiques. La plus utilisée est la méthode cytogénétique de mesure du nombre moyen d'aberrations chromosomiques portées par les lymphocytes circulants, qui permet une estimation de la dose globale reçue (AIEA, 2001), mais ne rend pas compte de l'hétérogénéité de l'irradiation. Pour tenter de palier à cet inconvénient, des modèles mathématiques ont été utilisés pour estimer l'hétérogénéité de l'irradiation en se basant sur la distribution du nombre d'aberrations par cellule (Sasaki et Miyata, 1968 ; Dolphin, 1969). Une amélioration considérable a été apportée par l'application de la cytogénétique à d'autres types cellulaires comme les fibroblastes de la peau autour de la zone irradiée (Kanda et al., 2002). Ces progrès ont permis de faire une cartographie dosimétrique des lésions cutanées à la suite d'un accident survenu en Géorgie (Pouget et al., 2004). Cependant, cette approche cytogénétique reste insuffisante pour une estimation fiable des dommages à la moelle osseuse.

Une autre approche est basée sur le concept de bio-indicateur d'atteinte. Il s'agit d'un paramètre biologique dont la variation renseigne sur la sévérité des dommages induits à la fonction physiologique ou à l'organe auquel il est associé 


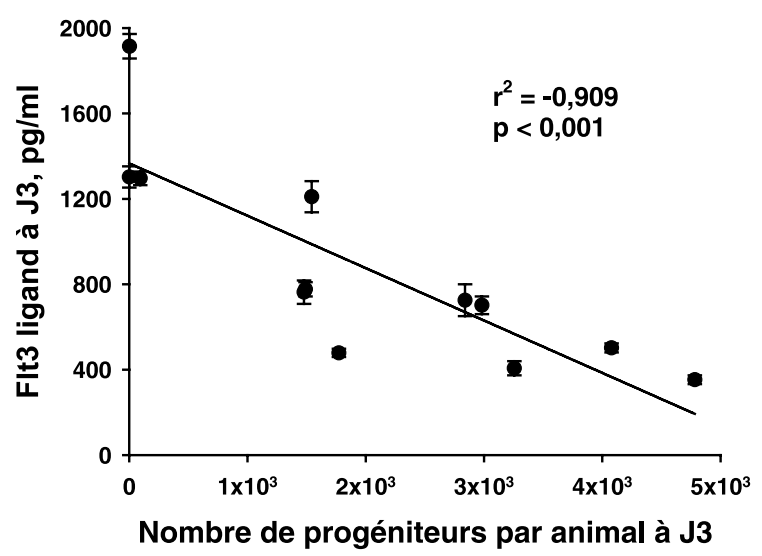

Figure 2-Analyse par régression linéaire de la corrélation négative existant entre la concentration plasmatique de Flt3 ligand et le nombre de progéniteurs hématopoḯtiques survivants dans la moelle osseuse au $3^{e}$ jour après irradiation. Cette corrélation a été obtenue chez des souris irradiées par une source de cobalt-60 soit corps entier soit de façon hétérogène (incluant $25 \%$, $50 \%$ ou $75 \%$ de la moelle osseuse), et à trois doses différentes (4, 7,5 et 11 Gy). Chaque point représente la moyenne \pm écart-type de 6 souris.

Analysis by linear regression of the correlation between plasma Flt3 ligand concentration and the number of surviving haematopoietic progenitors on day 3 after irradiation. This correlation was obtained in mice irradiated with a cobalt-60 source, with three different radiation doses (4, 7.5 and $11 \mathrm{~Gy})$ and with either a homogeneous irradiation or a heterogeneous irradiation (including $25 \%$, $50 \%$ or $75 \%$ of bone marrow). Each point represents the mean $\pm S D$ of 6 animals.

(Dublineau et al., 2004). Ainsi, l'irradiation des glandes salivaires entraîne une augmentation dose dépendante de l'amylase sérique (Barrett et al., 1982). Bien que les glandes parotides ne soient pas, loin s'en faut, un organe vital pour la survie de l'individu, une augmentation de l'amylase sérique en association avec d'autres bio-indicateurs plus généraux comme la dosimétrie par cytogénétique ou la pente de chute des lymphocytes peut renseigner sur l'hétérogénéité de l'irradiation (Silini et Gouskova, 1991). D'autres bio-indicateurs ont été mis en évidence ces dernières années, notamment la citrulline comme bio-indicateur de l'atteinte de la muqueuse intestinale (Lutgens et al., 2004), et le Flt3 ligand comme bio-indicateur de l'atteinte à la moelle osseuse (Prat et al., 2005, 2006). Il a été montré que l'augmentation de concentration plasmatique de Flt3 ligand est directement corrélée au nombre de progéniteurs hématopoḯtiques survivants dans la moelle osseuse, quelle que soit la dose d'irradiation (Prat et al., 2005) (Fig. 2). Cette corrélation donne un accès direct à une mesure de l'hématopoïèse résiduelle chez la victime d'irradiation accidentelle. 
L'intérêt majeur de ces deux bio-indicateurs est bien leur spécificité de tissus, indépendamment de la nature de l'agent inducteur du dommage. La concentration sanguine de Flt3 ligand est augmentée en cas d'aplasie, que ce soit à la suite d'une chimiothérapie ou d'une irradiation (Prat et al., 2006). Ces bio-indicateurs spécifiques d'une fonction physiologique complètent l'approche multiparamétrique intégrant la dosimétrie physique, la dosimétrie par cytogénétique, les signes cliniques du syndrome initial et les mesures de paramètres biochimiques et permettant de grader la sévérité d'une irradiation accidentelle (Fliedner et al., 2001; Roy et al., 2005). Le choix de la stratégie thérapeutique peut alors être basé sur l'estimation des dommages radio-induits aux organes ou aux fonctions physiologiques et non sur la dose moyenne reçue par la victime. Une telle approche multiparamétrique tient compte non seulement des particularités de l'accident (débit de dose, nature du rayonnement, hétérogénéité) mais aussi de la radiosensibilité individuelle.

\section{Les voies thérapeutiques possibles du syndrome hématopoïétique}

L'estimation des dommages radio-induits à la moelle osseuse plutôt que la dose moyenne à l'organisme est particulièrement cruciale pour le syndrome hématopoïétique, car en fonction de cette estimation, deux stratégies thérapeutiques peuvent être proposées : soit l'attente de la reprise hématopoïétique endogène à partir de la moelle osseuse résiduelle, soit le remplacement de la moelle osseuse lésée par une greffe.

La stratégie d'attente de la reprise endogène repose sur des traitements de soutien, avec une antibiothérapie préventive des infections opportunistes, des transfusions de plaquettes pour la réduction des risques hémorragiques, éventuellement en association avec des injections de cytokines capables de stimuler l'hématopoï̀se résiduelle, comme le granulocyte-colony stimulating factor (G-CSF) ou l'érythropoḯtine (EPO). C'est cette ligne de conduite thérapeutique qui a été recommandée par un collège d'experts français pour le traitement des victimes d'irradiation accidentelle (Jouet et al., 2003). L'efficacité du traitement par injection de cytokines n'a pu être démontrée formellement que dans des modèles animaux (Mac Vittie et Farese, 1995), et seul un parallèle avec la correction des neutropénies consécutives à un traitement aplasiant permet de suggérer un effet thérapeutique majeur chez les irradiés accidentels (Moulder, 2004). Il est à noter que ces deux cytokines sont à l'heure actuelle les seules molécules ayant reçu une autorisation de mise sur le marché (AMM) pour ce type d'application. L'intérêt de cette stratégie d'attente est le fait qu'elle permet une réévaluation régulière de son bien-fondé en recherchant les signes d'une sortie 
d'aplasie, tout en permettant la préparation d'une greffe de moelle osseuse si besoin est. En effet, la greffe sera dans la très grande majorité des cas allogénique, c'est-à-dire issue d'un donneur compatible mais génétiquement différent. Ce type de greffe nécessite la recherche d'un donneur compatible, ce qui peut prendre plusieurs semaines. De plus, la greffe allogénique nécessite la préparation immunologique du receveur de façon à outrepasser les barrières immunitaires et éviter les réactions du greffon contre l'hôte $(\mathrm{GVH})$ ou de rejet de greffe (Dainiak, 2005), ces deux réactions pouvant être délétères au point d'engager le pronostic vital des patients (Ringden et al., 2005). Malgré ces risques élevés associés à la greffe de CSH, un concept de greffe transitoire a été proposé (Dainiak, 2005) de façon à protéger le patient des hémorragies et des infections avec un apport de cellules fonctionnelles en attendant une reprise endogène. C'est ce qui a été réalisé chez l'une des victimes de l'accident de Tokai-mura (Nagayama et al., 2002). Cependant, le bilan du traitement des victimes d'irradiation accidentelles montre que la greffe de moelle osseuse représente une thérapie à risques. Au total, 31 victimes d'irradiation ont reçu une greffe de $\mathrm{CSH}$, quelle que soit l'origine des cellules : moelle osseuse, sang périphérique mobilisé, sang de cordon ombilical ou foie fœtal. Seuls quatre patients ont survécu plus de 6 mois. Une étude rétrospective réalisée en 1997 (et donc n'incluant pas les victimes de Tokai-mura) a comparé le devenir des victimes greffées avec des $\mathrm{CSH}$ par rapport à des victimes traitées uniquement par thérapie de soutien (Densow et al., 1997). Vingt-neuf victimes ont été inclues dans chaque groupe, avec une correspondance établie uniquement pour la dose d'irradiation (Fig. 3). Étant donné le faible nombre de patients dans chaque groupe, et donc l'existence possible de biais liés à d'autres paramètres comme l'âge, le sexe, les circonstances de l'accident ou la cause du décès, cette étude comparative doit être prise avec beaucoup de précautions. Néanmoins, les résultats (Fig. 3) suggèrent que la survie à 6 mois est en faveur du groupe de patients non greffés ( $24 \%$ vs. $14 \%$ dans le groupe de patients greffés).

Globalement, l'application de la greffe de $\mathrm{CSH}$ reste donc une option à risques élevés pour le patient. Ceci a motivé la recherche de nouvelles options thérapeutiques ces dernières années, et notamment la possibilité d'appliquer la thérapie cellulaire autologue au cas des irradiations accidentelles.

\section{Apports et limites de la thérapie cellulaire autologue appliquée aux cellules hématopoiétiques}

Le principe de la thérapie cellulaire autologue est d'utiliser les cellules hématopoïétiques survivantes du patient pour en amplifier le nombre in vitro, puis les réinjecter au moment opportun. De nombreux protocoles de culture cellulaire et de réinjection ont été décrits, et montrent qu'il est possible d'amplifier des 


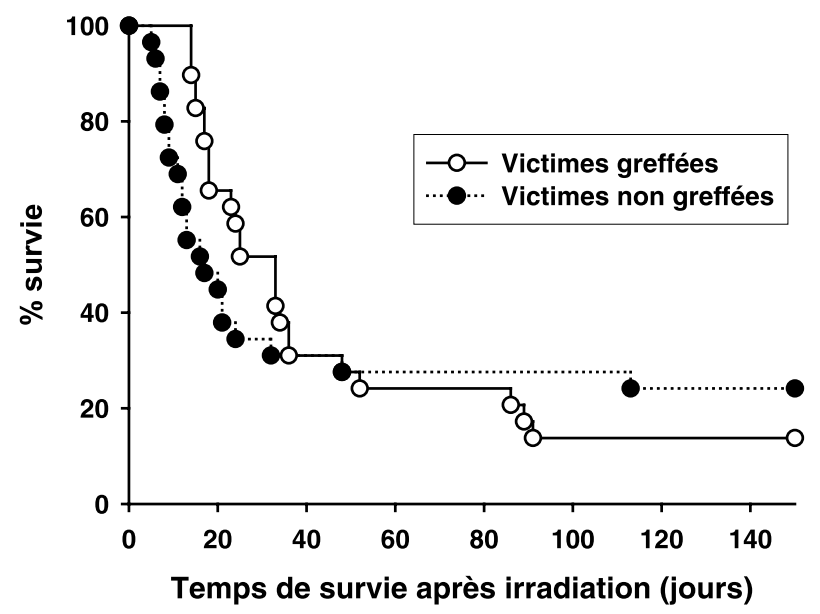

Figure 3 - comparaison de la survie à long terme des victimes d'irradiation traitées (ronds blancs) ou non (ronds noirs) par greffe de CSH. D'après les données de Densow et al. (1997).

Comparison of long term survival of irradiated victims that were treated either with (open circles) or without (closed circles) haematopoietic stem cell transplantation. According to the data from Densow et al. (1997).

cellules hématopoḯtiques humaines en quantités et de la nature souhaitée en fonction des besoins du patient (Hoffman, 1999). De plus, les cellules produites conservent leur potentiel thérapeutique. Différentes études chez l'homme et chez l'animal ont montré que la réinjection, après chimiothérapie ou après irradiation, de cellules produites par amplification ex vivo à partir d'un prélèvement fait avant le traitement aplasiant, permet d'obtenir des réduction importantes de profondeur et de durée d'aplasie (Paquette et al., 2000 ; Bertho et al., 2002), et des essais cliniques sont déjà en cours pour confirmer ces observations (Jaroscak et al., 2003).

L'application de la thérapie cellulaire autologue à la situation d'irradiation accidentelle a été proposée récemment (Hérodin et Drouet, 2002). Elle présente l'avantage majeur d'être réalisable dans un contexte autologue, c'est à dire sans risque de rejet ou de réaction de GVH. Cependant, elle est basée sur deux hypothèses. La première est l'existence d'une hématopoï̀se résiduelle, due à l'hétérogénéité de l'irradiation accidentelle. Ceci a largement été démontré dans des modèles animaux aussi bien que chez l'homme (Parmentier et al., 1980; Baranov et al., 1994). La seconde hypothèse est qu'il est possible d'amplifier in vitro et de façon efficace des cellules hématopoïétiques irradiées. Ceci a été vérifié par plusieurs groupes, soit à partir de CSH enrichies (Drouet et al., 1999), soit 
à partir de cellules hématopoïétiques totales (Bertho et al., 2004). Cependant, l'efficacité de l'amplification ex vivo des cellules hématopoïétiques, tant quantitative que qualitative, est directement corrélée à la dose d'irradiation reçue par ces cellules, et une réduction de moitié du facteur d'amplification des cellules hématopoïétiques est observée dès la dose de 2 Gy (Bertho et al., 2004).

Deux essais de thérapie cellulaire autologue ont été réalisés chez le primate non humain, pour vérifier la faisabilité de ce type de protocole. Le premier essai a été réalisé chez le babouin après irradiation globale homogène à 6 Gy (Hérodin et Drouet, 2002). Les résultats ont montré que les CSH peuvent être recueillies par cytaphérèse dans le sang périphérique, puis amplifiées ex vivo dans les 24 heures suivant l'irradiation. Cependant, le nombre de CSH recueillies (environ $1 \times$ $10^{6}$ cellules par litre de cytaphérèse) et leur capacité d'expansion restent faibles, d'où une efficacité thérapeutique limitée à une réduction de durée de la neutropénie, mais sans effet sur une thrombopénie sévère, ce qui a conduit au décès des animaux (Hérodin et Drouet, 2002).

Le second essai a été réalisé chez le macaque, avec un système d'irradiation hétérogène, où un bras a été partiellement protégé de l'irradiation. Il en résulte une irradiation corporelle globale à la dose de $8 \mathrm{~Gy}$ et laissant un volume de $4,5 \%$ de la moelle osseuse ayant reçu une dose de 3,5 Gy (Bertho et al., 2005a). Ces conditions d'irradiation peuvent être considérées comme représentatives d'un accident d'irradiation globale hétérogène à forte dose. Dans ce modèle, la moelle osseuse a été prélevée directement dans le bras partiellement protégé dans les deux heures suivant l'irradiation et mise en culture immédiatement. Là encore, l'efficacité thérapeutique des cellules amplifiées ex vivo est limitée à une réduction de la profondeur de la neutropénie et de la thrombopénie, en corrélation avec la dose d'irradiation reçue par le bras protégé. Cependant, un point intéressant de cette étude est que chez la plupart des animaux, y compris le groupe témoin positif traité par expansion faite à partir de cellules non irradiées, une pathologie sévère est apparue au cours de la troisième semaine après irradiation. Cette pathologie, assimilée à un syndrome de défaillance multi-viscérale (SDMV), a impliqué non seulement le système hématopoiétique, mais aussi le tractus digestif et les fonctions rénales et hépatiques, avec une suspicion de syndrome de coagulation intra-vasculaire disséminée (Bertho et al., 2005a). Ce SDMV radio-induit a par ailleurs déjà été observé chez l'une des victimes de l'accident de Tokai-mura (Asano, 2005), mais aussi dans d'autres accidents d'irradiation (Konchalovsky et al., 2005), et constitue à l'heure actuelle un mur thérapeutique. Un modèle d'irradiation hétérogène à forte dose tel que celui développé chez le macaque en protégeant partiellement un territoire médullaire, avec un support hématologique éventuellement apporté par un protocole de thérapie cellulaire autologue, pourrait constituer un modèle d'étude de ce SDMV radio-induit. 


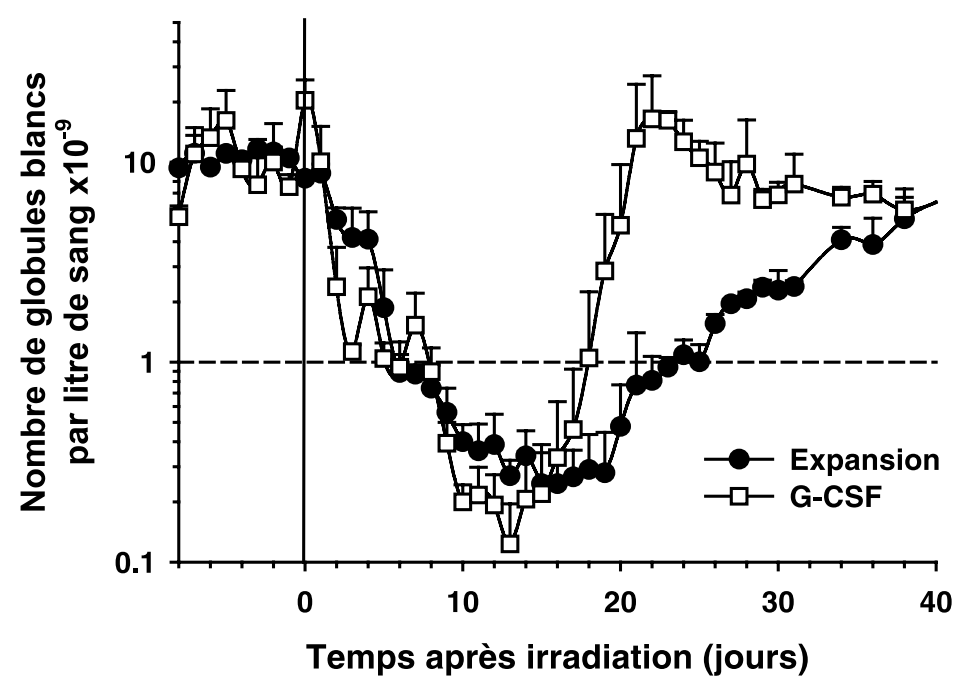

Figure 4-Comparaison de l'évolution du nombre de globules blancs en fonction du temps après irradiation entre un groupe d'animaux traités par G-CSF seul (carrés blancs, $n=3$ ) et un groupe traité par expansion ex vivo seule (cercles pleins, $n=3$ ). Les résultats sont donnés en moyenne \pm écart-type. La ligne en pointillée représente la limite de risque d'apparition d'infections opportunistes. D'après les données de Bertho et al. (2005a, 2005b).

Comparison of white blood cell number variations according to the time after irradiation between a group of animals treated with $G-C S F$ alone (open squares, $n=3$ ) and a group of animals treated with ex vivo expanded haematopoietic cells alone (closed circles, $n=3$ ). Results are given as mean $\pm S D$. The dotted line indicates the limit for the appearance of opportunistic infections. According to the data from Bertho et al. (2005a, 2005b).

\section{La thérapie par injections de G-CSF}

Une autre étude a été réalisée chez le macaque dans le même modèle d'irradiation hétérogène, en combinant la thérapie cellulaire autologue avec des injections de G-CSF. Les injections de G-CSF ont été récemment préconisées pour le traitement des victimes d'irradiation accidentelle (Jouet et al., 2003). Les résultats ont montré une très grande efficacité du G-CSF pour stimuler l'hématopoïèse résiduelle et la sortie d'aplasie, que ce soit en combinaison ou non avec la thérapie cellulaire autologue. De plus, le suivi de l'ensemble des paramètres sanguins et médullaires n'a montré aucune différence entre les animaux traités par G-CSF et ceux traités par G-CSF et thérapie cellulaire (Bertho et al., 2005b), suggérant que la thérapie cellulaire n'apporte pas de bénéfice thérapeutique par rapport à un traitement par G-CSF seul. Ceci est confirmé par la comparaison des résultats entre les deux séries d'expériences (Fig. 4), qui montre que les animaux traités par G-CSF seul 
sont en phase de sortie d'aplasie au moment où les animaux traités par thérapie cellulaire seule sont encore en aplasie profonde. Ces résultats sont la démonstration expérimentale de la validité de l'approche thérapeutique proposée par un groupe de cliniciens, qui consiste à stimuler l'hématopoï̀se résiduelle par des injections de G-CSF aussi tôt que possible après l'accident d'irradiation, et à retarder la décision de greffe de CSH (Jouet et al., 2003).

Globalement, les résultats obtenus avec la thérapie cellulaire autologue appliquée à un modèle d'irradiation accidentelle hétérogène se sont avérés décevants, du fait essentiellement de la faible capacité de prolifération des cellules hématopoiétiques irradiées. Cependant, ceci ne remet pas en cause la thérapie par expansion ex vivo autologue, si elle est appliquée au traitement des effets secondaires des thérapies anti-cancéreuses. De plus, les principes de thérapie cellulaire autologue peuvent être appliqués à des irradiations localisées. Cette approche de thérapie cellulaire autologue est particulièrement prometteuse dans le cas des brûlures radiologiques, pour lesquels il est envisageable d'utiliser non seulement des cellules épithéliales autologues pour des greffes de peau, mais également des greffes de cellules souches mésenchymateuses pour la reconstruction des tissus sous-jacents.

\section{Conclusion : revisiter la notion d'hématopoièse résiduelle, une voie d'avenir?}

Comme on peut le voir, la notion centrale à prendre en compte dans le choix d'une thérapie pour le syndrome hématopoïétique est la notion d'hématopoïèse résiduelle. La description récente du Flt3 ligand plasmatique comme bioindicateur de l'atteinte à la moelle osseuse représente un progrès important dans l'évaluation du syndrome hématopoiétique dans un contexte de diagnostic multiparamétrique. Cependant, ce bio-indicateur ne permettra pas de détecter une hématopoïèse résiduelle très réduite. Les exemples sont nombreux où l'existence de quelques CSH résiduelles, survivantes à une irradiation à forte dose, sont capables de reconstituer une hématopoï̀se endogène. La comparaison des différentes approches thérapeutiques proposées (Tab. I) montre que les stratégies d'attente (traitement de soutien et injection de cytokines) présentent des avantages importants du fait de leur réversibilité et de l'absence de risques de GVHD et de rejet par rapport à une greffe de CSH. D'autre part, la thérapie cellulaire, malgré l'avantage du contexte autologue, a montré une efficacité limitée, dépendante de la dose d'irradiation reçue par les cellules recueillies. Nos résultats récents sur le traitement par G-CSF démontrent que la stimulation de ces cellules résiduelles peut favoriser une reconstitution rapide, efficace et à long terme, ce qui apporte un avantage supplémentaire aux traitements de soutien (Tab. I). Ces résultats 
TABLEAU I

Analyse comparée des différentes approches thérapeutiques possibles du syndrome hématopoïétique induit par une irradiation accidentelle.

Comparative analysis of different therapeutic approaches for the treatment of occidental radiation-induced hematopoietic syndrome.

\begin{tabular}{|c|c|c|c|}
\hline $\begin{array}{c}\text { Approche } \\
\text { thérapeutique }\end{array}$ & Condition d'application & Avantages & Inconvénients \\
\hline $\begin{array}{c}\text { Soutien } \\
\text { (transfusions, } \\
\text { antibiothérapie) }\end{array}$ & $\begin{array}{l}\text { - Irradiation hétérogène } \\
\text { - Évidence d'hématopoï̀se } \\
\text { résiduelle }\end{array}$ & - Option révisable & $\begin{array}{l}\text { - Risques infectieux et } \\
\text { hémorragiques } \\
\text { - Aplasie de longue durée }\end{array}$ \\
\hline $\begin{array}{c}\text { Cytokines } \\
\text { (G-CSF, EPO) }\end{array}$ & $\begin{array}{l}\text { - Irradiation hétérogène } \\
\text { - Évidence d'hématopoï̀se } \\
\text { résiduelle }\end{array}$ & $\begin{array}{l}\text { - Option révisable } \\
\text { - Risques infectieux et } \\
\text { hémorragiques réduits } \\
\text { - Réduction de la durée d'aplasie }\end{array}$ & $\begin{array}{l}\text { - Nombre limité de } \\
\text { cytokines disponibles } \\
\text { (EPO, G-CSF) } \\
\text { - Efficacité prouvée par } \\
\text { analogie }\end{array}$ \\
\hline Greffe de CSH & $\begin{array}{l}\text { - Irradiation homogène } \\
\text { - Absence d'hématopoï̀se } \\
\text { résiduelle }\end{array}$ & $\begin{array}{l}\text { - Reconstitution à long terme } \\
\text { - Apport de cellules non irradiées }\end{array}$ & $\begin{array}{l}\text { - Recherche d'un donneur } \\
\text { compatible } \\
\text { - Risques de GVHD et de } \\
\text { rejet } \\
\text { - Nécessité de traitement } \\
\text { immunosuppresseur }\end{array}$ \\
\hline $\begin{array}{l}\text { Thérapie } \\
\text { cellulaire } \\
\text { autologue }\end{array}$ & $\begin{array}{l}\text { - Irradiation hétérogène } \\
\text { - Évidence d'hématopoï̀se } \\
\text { résiduelle } \\
\text { - Possibilité de prélèvement }\end{array}$ & $\begin{array}{l}\text { - Apport de cellules } \\
\text { fonctionnelles } \\
\text { - Système autologue }\end{array}$ & $\begin{array}{l}\text { - Effet à long terme? } \\
\text { - Efficacité dépendante de } \\
\text { la dose d'irradiation } \\
\text { - Traitement expérimental }\end{array}$ \\
\hline
\end{tabular}

constituent la démonstration expérimentale de la validité de l'approche thérapeutique recommandée par un collège d'experts en 2003 (Jouet et al., 2003) puis par un groupe de travail européen réuni en octobre 2005 sous la houlette de l'European group for Blood and Marrow Transplantation (EBMT) et de l'IRSN. Cette approche consiste à retarder la décision de greffe le plus possible dans l'attente des signes de reprise hématopoïétique endogène et à apporter un traitement de support avec injections de G-CSF le plus tôt possible après irradiation. Cependant, les mécanismes d'action du G-CSF et de régulation de l'hématopoï̀se résiduelle après irradiation restent mal connus. Ceci suggère que de nouvelles améliorations dans le traitement du syndrome hématopoḯtique radioinduit viendront d'une réévaluation de cette notion d'hématopoï̀se résiduelle, avec les outils et les moyens de la biologie actuelle.

Remerciements. L'auteur tient particulièrement à remercier P. Monti, N. Dudoignon, J. Aigueperse et P. Gourmelon pour les discussions fructueuses et la lecture critique du manuscrit, M. Prat, J. Frick et C. Demarquay pour leur collaboration précieuse dans la réalisation des expériences, et $R$. Riou et son équipe pour les soins quotidiens aux animaux. Ce travail a été soutenu financièrement par Électricité de France et Chugai Pharma France. 


\section{RÉFÉRENCES}

AIEA (2001) Cytogenetic analysis for dose assessment: A manual. Technical reports series $\mathrm{N}^{\circ} 405$, AIEA, Vienna.

Asano S. (2005) Multi-organ involvement: Lessons from the experience of one victim of the Tokaimura criticality accident, Br. J. Radiol. 27(sup), 9-12.

Baranov A.E., Selidovkin G.D., Butturini A., Gale R.P. (1994) Hematopoietic recovery after 10 Gy acute total body radiation, Blood 83, 596-599.

Barrett A., Jacobs A., Kohn J., Raymond J., Powles R.L. (1982) Changes in serum amylase and its isoenzymes after whole body irradiation, Br. Med. J. 285, 170-171.

Bertho J.M., Frick J., Demarquay C., Lauby A., Mathieu E., Dudoignon N., Jacquet N., Chau Q., Joubert C., Chapel A., Lopez M., Aigueperse J., Gorin N.C., Gourmelon P., Thierry D. (2002) Reinjection of ex vivo expanded primate bone marrow mononuclear cells strongly reduces radiation-induced aplasia, J. Hematother. Stem Cell Res. 11, 549-564.

Bertho J.M., Mathieu E., Lauby A., Frick J., Demarquay C., Gourmelon P., Gorin N.C., Thierry D. (2004) Feasibility and limits of bone marrow mononuclear cell expansion following irradiation, Int. J. Radiat. Biol. 80, 73-81.

Bertho J.M., Prat M., Frick J., Demarquay C., Gaugler M.H., Dudoignon N., Clairand I., Chapel A., Gorin N.C., Thierry D., Gourmelon P. (2005a) Application of autologous hematopoietic cell therapy to a non human primate model of heterogeneous high dose irradiation, Radiat. Res. 163, $557-570$.

Bertho J.M., Frick J., Prat M., Demarquay C., Dudoignon N., Trompier F., Gorin N.C., Thierry D., Gourmelon P. (2005b) Comparison of autologous cell therapy and granulocyte-colony stimulating factor (G-CSF) injection $v s$. G-CSF injection alone for the treatment of acute radiation syndrome in a non human primate model, Int. J. Radiat. Oncol. Biol. Phys. 63, 911920.

Cristy M. (1981) Active bone marrow distribution as a function of age in humans, Phys. Med. Biol. 26, $389-400$.

Dainiak N. (2005) The evolving role of haematopoietic cell transplantation in radiation injury: potential and limitations, Br. J. Haematol. 27(sup), 169-174.

Densow D., Kindler H., Baranov A.E., Tibken B., Hofer E.P., Fliedner T.M. (1997) Criteria for the selection of radiation accident victims for stem cell transplantation, Stem Cells 15(sup2), 287297.

Dolphin G.W. (1969) Biological dosimetry with particular reference to chromosome aberration analysis, A review of methods, Handling of radiation accidents, pp. 215-224. AIEA, Vienne.

Drouet M., Mathieu J., Grenier N., Multon E., Sotto J.J., Herodin F. (1999) The reduction of in vitro radiation-induced fas-related apoptosis in $\mathrm{CD} 34^{+}$progenitor cells by SCF, Flt-3 ligand, TPO, and IL-3 in combination resulted in CD $34^{+}$cell proliferation and differentiation, Stem Cells 17, 273-285.

Dublineau I., Dudoignon N., Monti P., Combes O., Wysocki J., Grison S., Baudelin C., Griffiths N.M., Scanff P. (2004) Screening of a large panel of gastrointestinal peptide plasma levels is not adaptated for the evaluation of digestive damage following irradiation, Can. J. Physiol. Pharmacol. 82, 103-113.

Fliedner T.M., Friesecke I., Beyrer K. (Eds) (2001) The medical management of radiation accidents, manual on the acute radiation syndrome. British Institute of Radiology, London.

Hérodin F., Drouet M. (2002) Autologous cell therapy as a new approach to treatment of radiationinduced bone marrow aplasia: preliminary study in a baboon model, Can. J. Physiol. Pharmacol. 80, 710-716. 
Hoffman R. (1999) Progress in the development of systems for in vitro expansion of human hematopoietic stem cells, Curr. Opin. Hematol. 6, 184-191.

Jaroscak J., Goltry K., Smith A., Waters-Pick B., Martin P.L., Driscoll T.A., Howrey R., Chao N., Douville J., Burhop S., Fu P., Kurtzberg J. (2003) Augmentation of umbilical cord blood transplantation with ex vivo-expanded umbilical cord blood cells: Results of a phase I trial using the AastromReplicell system, Blood 101, 5061-5067.

Jouet J.P., Gorin N.C., Gourmelon P. (2003) Gestion médicale des victimes d'irradiation lors d'un accident nucléaire ou d'un acte de malveillance de grande ampleur, Les entretiens de Bichat, tables rondes thérapeutique, $p p$. 144-147. Expansion scientifique française, Paris.

Kanda R., Minamihisamatsu M., Hayata I. (2002) Dynamic analysis of chromosome aberrations in three victims of the Tokai-Mura criticality accident, Int. J. Radiat. Biol. 78, 857-862.

Konchalovsky M.V., Barabov A.E., Kolganov A.V. (2005) Multiple organ involvement and failure: Selected Russian radiation accident cases re-visited, Br. J. Radiol. 27(sup), 26-29.

Lutgens L.C., Deutz N., Granzier-Peeters M., Beets-Tan R., De Ruysscher D., Gueulette J., Cleutjens J., Berger M., Wouters B., von Meyenfeldt M., Lambin P. (2004) Plasma citrulline concentration: A surrogate end point for radiation-induced mucosal atrophy of the small bowel. A feasibility study in 23 patients, Int. J. Radiat. Oncol. Biol. Phys. 60, 275-285.

Mac Vittie T.J., Farese A.M. (1995) Experimental approaches for therapeutic treatment of radiationinduced haemopoietic injury, Radiation toxicology, bone marrow and leukemia (J.H. Hendry et B.I. Lord, Eds), pp. 141-193. Taylor \& Francis, Londres.

Moulder J.E. (2004) Post-irradiation approaches to the treatment of radiation injuries in the context of radiological terrorism and radiation accidents: a review, Int. J. Radiat. Biol. 80, 3-10.

Nagayama H., Misawa K., Tanaka H., Ooi J., Iseki T., Tojo A., Tani K., Yamada Y., Kodo H., Takahashi T.A., Yamashita N., Shimazaki S., Asano S. (2002) Transient haematopoietic stem cell rescue using umbilical cord blood for a lethally irradiated nuclear accident victim, Bone Marrow Transpl. 29, 197-204.

Paquette R.L., Dergham S.T., Karpf E., Wang H.J., Slamon D.J., Souza L., Glaspy J.A. (2000) Ex vivo expanded unselected peripheral blood: Progenitor cells reduce posttransplantation neutropenia, thrombocytopenia, and anemia in patients with breast cancer, Blood 96, 2385-2390.

Parmentier N.C., Nénot J.C., Jammet H.J. (1980) A dosimetric study of the Belgian (1965) and Italian (1975) accidents, The medical basis for radiation accident preparedness (K.F. Hübner, S.A. Fry, Eds) pp. 105-112. Elsevier, Amsterdam.

Pouget J.P., Laurent C., Delbos M., Benderitter M., Clairand I., Trompier F., Stephanazzi J., Carsin H., Lambert F., Voisin P., Gourmelon P. (2004) PCC-FISH in skin fibroblasts for local dose assessment: biodosimetric analysis of a victim of the Georgian radiological accident, Radiat. Res. 162, 365-376.

Prat M., Demarquay C., Frick J., Thierry D., Gorin N.C., Bertho J.M. (2005) Radiation induced increase in plasma Flt3-ligand concentration in mice: evidence for the implication of several cell types, Radiat. Res. 163, 408-417.

Prat M., Frick J., Laporte J.-Ph., Thierry D., Gorin N.C., Bertho J.M. (2006) Kinetics of plasma Flt3 ligand concentration in haematopoietic stem cell transplanted patients, Leuk. Lymph. 47, 77-80.

Ringden O.T.H., Le Blanc K., Remberger M. (2005) Granulocyte and granulocyte-macrophage colonystimulating factors in allografts: Uses, misuses, misconceptions and future applications, Exp. Hematol. 33, 505-512.

Roy L., Bertho J.M., Souidi M., Vozenin M.C., Voisin Ph., Benderitter M. (2005) Biochemical approach to prediction of multiple organ dynsfunction syndrome, Br. J. Haematol. 27(sup), 146151.

Sasaki M.S., Miyata H. (1968) Biological dosimetry in atomic bomb survivors, Nature 220, 1189-1193.

Silini G., Gouskova A. (1991) Biological dosimetry at Chernobyl, New horizons in biological dosimetry, (B.L. Gledhill, F. Mauro, Eds) pp. 129-144. Wiley-Liss, New York. 